\title{
Identification of Mild Cognitive Impairment Subtypes Predicting Conversion to Alzheimer's Disease Using a Heterogeneous Mixture Learning
}

Masataka Kikuchi ( $\square$ kikuchi@gi.med.osaka-u.ac.jp )

Osaka University School of Medicine Graduate School of Medicine: Osaka Daigaku Daigakuin Igakukei Kenkyuka Igakubu https://orcid.org/0000-0002-9562-841X

Kaori Kobayashi

Osaka University

Sakiko Itoh

Osaka University

Kensaku Kasuga

Niigata University

Akinori Miyashita

Niigata University

Takeshi Ikeuchi

Niigata University

Eiji Yumoto

NEC corporation

Yasuto Fushimi

NEC corporation

Toshihiro Takeda

Osaka University School of Medicine Graduate School of Medicine: Osaka Daigaku Daigakuin Igakukei Kenkyuka Igakubu

\section{Shirou Manabe}

Osaka University School of Medicine Graduate School of Medicine: Osaka Daigaku Daigakuin Igakukei Kenkyuka Igakubu

\section{Kenichi Kamijo}

NEC corporation

\section{Yasushi Matsumura}

Osaka University School of Medicine Graduate School of Medicine: Osaka Daigaku Daigakuin Igakukei Kenkyuka Igakubu 
Keywords: Alzheimer Disease, Mild Cognitive Impairment, Decision Trees

Posted Date: December 17th, 2020

DOI: https://doi.org/10.21203/rs.3.rs-129455/v1

License: (c) (i) This work is licensed under a Creative Commons Attribution 4.0 International License. Read Full License 
1 Identification of mild cognitive impairment subtypes predicting

2 conversion to Alzheimer's disease using a heterogeneous mixture

3 learning

4

5 Masataka Kikuchi ${ }^{1}$, Kaori Kobayashi ${ }^{1,2}$, Sakiko Itoh $^{1}$, Kensaku Kasuga ${ }^{3}$, Akinori

6 Miyashita $^{3}$, Takeshi Ikeuchi ${ }^{3}$, Eiji Yumoto ${ }^{4}$, Yasuto Fushimi ${ }^{2}$, Toshihiro Takeda ${ }^{5}$, Shirou

7 Manabe ${ }^{5}$, Alzheimer's Disease Neuroimaging Initiative ${ }^{\#}$, Kenichi Kamijo ${ }^{2}$, Yasushi

8 Matsumura $^{5}$

9

$10{ }^{1}$ Department of Genome Informatics, Graduate School of Medicine, Osaka University,

11 Osaka, Japan

$12{ }^{2}$ Medical Solutions Division, NEC Corporation, Tokyo, Japan

$13{ }^{3}$ Department of Molecular Genetics, Brain Research Institute, Niigata University,

14 Niigata, Japan

$15 \quad{ }^{4}$ Biometrics Research Laboratories, NEC Corporation, Tokyo, Japan

$16{ }^{5}$ Department of Medical Informatics, Graduate School of Medicine, Osaka University,

17 Osaka, Japan

18 " The data used in preparation of this article were obtained from the Alzheimer's

19 Disease Neuroimaging Initiative (ADNI) database (adni.loni.usc.edu). As such, the

20 investigators within the ADNI contributed to the design and implementation of the

21 ADNI and/or provided data but did not participate in the analysis or writing of this

22 report. A complete listing of ADNI investigators can be found at

23 http://adni.loni.usc.edu/wp-content/uploads/how_to_

24 apply/ADNI_Acknowledgement_List.pdf.

To whom correspondence should be addressed:

Masataka Kikuchi

2-2 Yamadaoka, Suita, Osaka 565-0871, Japan

29

Phone No.: +81-6-6210-8363

Fax No.: +81-6-6210-8365

Email Address: kikuchi@gi.med.osaka-u.ac.jp (M.K.) 


\section{Abstract}

\section{Background}

4 dementias, including Alzheimer's disease (AD) dementia. However, individuals with

6 AD dementia. Detailed subtyping of MCI and accurate prediction of the patients in whom

7 MCI will convert to AD dementia may support new trial designs and enable evaluation

8 of the efficacy of drugs within small numbers of patients during clinical trials.

\section{Methods}

We constructed a decision tree model by the heterogeneous mixture learning (HML) method, integrating cerebrospinal fluid (CSF) biomarker data, structural MRI data, $A P O E$ genotype data, and a recorded age at examination. The decision tree model was applied to predict conversion to AD dementia and to identify subtypes of MCI. After the test performances of HML models were assessed, MCI subjects were classified into some subtypes based on a decision tree. Then, we characterized each MCI subtype in terms of the degree of CSF biomarker abnormalities and brain atrophy, declines of cognitive functions, and gene expression alterations derived from peripheral blood samples. 


\section{Results}

3 them into three groups: those similar to $\mathrm{CN}$ subjects with low conversion rates; those with

4 intermediate conversion rates; and those similar to patients with $\mathrm{AD}$ with high conversion

5 rates. Furthermore, the subtypes with intermediate conversion rates were separated into

6 the subtype with CSF biomarker abnormalities or the subtype with brain atrophy. The

7 results from the CSF inflammation marker and gene expression analysis suggested the

8 occurrence of aberrant inflammatory immune responses in the CSF and blood of the

9 subjects in the subtypes with CSF biomarker abnormalities.

\section{Conclusion}

to $\mathrm{AD}$ as well as differing levels of biological features. Focusing on specific subtypes in which conversion to $\mathrm{AD}$ can be predicted with the most accuracy could enable more efficient clinical trials to be conducted.

\section{Keywords:}

17 Alzheimer Disease, Mild Cognitive Impairment, Decision Trees 


\section{Background}

3 dementia, which is characterized by the deposition of amyloid-beta $(\mathrm{A} \beta)$ protein and tau

4 protein [1]. Overcoming dementia is an urgent issue because the number of affected

5 individuals is increasing.

7 people with $\mathrm{AD}$ in clinical trials or have shown adverse effects [2]. Although these drugs

8 do not demonstrate excellent clinical efficacy in patients with late-stage $\mathrm{AD}$, they can

9 potentially be effective for the treatment of patients with early-stage AD or MCI. However, individuals with MCI show heterogeneity in patterns of pathology, and MCI does not always convert to $\mathrm{AD}$ dementia. Detailed subtyping of MCI and accurate prediction of the patients in whom MCI will convert to $\mathrm{AD}$ dementia may support new trial designs and enable evaluation of the efficacy of drugs within small numbers of patients during clinical trials. divides MCI into amnestic and non-amnestic MCI, with further division into a group with a single impairment in the cognitive domain (single-domain MCI) and a group with multiple impairments (multiple-domain MCI). In particular, amnestic MCI, regardless of 
1 whether it is single- or multiple-domain MCI, converts to dementia, mainly AD dementia,

2 at a rate of $10 \%$ to $15 \%$ per year [4]. Recent studies based on neuropsychological tests

3 have also identified some subtypes of MCI [5, 6]. However, clinical diagnoses and

4 neuropsychological testing often include subjective factors. In addition to subjective

5 factors, assessment of objective factors such as brain imaging data, biomarker data, and

6 genomic data may enable more precise determination of a subtype of MCI that converts

7 to $\mathrm{AD}$ dementia.

8

In this study, we applied the HML method to identify subtypes of MCI. HML

9 divides individuals into similar groups based on the brain volumes from five brain regions,

10 CSF biomarker including $\mathrm{A} \beta$ and tau, and genomic data of apolipoprotein $\mathrm{E}(A P O E)$

11 gene obtained from individuals and generates appropriate predictive models for each

12 group (e.g., models for determining whether an individual is a patient with AD dementia

13 or a healthy individual). We characterized the subtypes of MCI identified by HML and

14 examined conversion to $\mathrm{AD}$ dementia for each subtype over a given period.

16 Materials and methods

\section{Dataset}

The data used in this study were obtained from the Alzheimer's Disease 
1 Neuroimaging Initiative (ADNI) [7]. The ADNI was launched in 2003 as a public-private

2 partnership led by Principal Investigator Michael W. Weiner, MD. The primary goal of

3 the ADNI has been to test whether serial magnetic resonance imaging (MRI), positron

4 emission tomography (PET), analysis of other biological markers, and clinical and

5 neuropsychological assessments can be combined to measure the progression of MCI and

6 early AD. The ADNI database contains data from a large number of cognitively normal

7 (CN), MCI, and AD subjects recruited from over 50 different centres in the US and

8 Canada, with follow-up assessments performed every 6 months. Institutional review

9 boards approved the study procedures across participating institutions. Written informed

10 consent was obtained from all subjects.

This study considered the data of 941 subjects comprising 156 AD dementia

patients (at baseline; same hereinafter), $480 \mathrm{MCI}$ subjects, and $305 \mathrm{CN}$ subjects. All

subjects had CSF biomarker data, structural MRI data, APOE genotype data, and a

recorded age at examination. The $\mathrm{AD}$ dementia patients and the MCI subjects were

diagnosed mainly by neuropsychological tests (Mini-Mental State Examination (MMSE),

Clinical Dementia Rating-Sum of Boxes (CDR-SB), and Wechsler Memory Scale

Logical Memory II). Based on the above criteria, the AD dementia patients that were 
1 clinically diagnosed without the pathological markers such as $A \beta$ and tau proteins. For

2 this study, we used a subset of the ADNI dataset called ADNIMERGE. Table 1 shows a

3 summary of each group.

4

\section{CSF biomarkers}

6 The CSF biomarkers comprised the following five markers: $A \beta(1-42)$ peptide

7 levels, total tau (tTau) protein levels, phosphorylated tau (pTau) protein levels, the $8 \mathrm{tTau} / \mathrm{A} \beta(1-42)$ ratio, and the $\mathrm{pTau} / \mathrm{A} \beta(1-42)$ ratio. The levels of $\mathrm{A} \beta(1-42), \mathrm{tTau}$, and $\mathrm{pTau}$

9 were analysed by Roche Elecsys ${ }^{\circledR}$ immunoassays (Roche Diagnostics GmbH, Penzberg, 10 Germany). The $\mathrm{tTau} / \mathrm{A} \beta(1-42)$ ratio and $\mathrm{pTau} / \mathrm{A} \beta(1-42)$ ratio were calculated by the levels

11 of the above three CSF biomarkers. The CSF biomarkers are quantitative variables, but 12 the level of each is often represented by a string containing an inequality sign when the 13 biomarker level reaches the upper limit or is below the detection limit of immunoassays.

14 Here, we treated ">1700" for $\mathrm{A} \beta(1-42)$ as $1,700 \mathrm{pg} / \mathrm{mL}$ and ">1300" for tTau as 1,300 pg/mL. Similarly, "<8" and ">120" for pTau were transformed into $8 \mathrm{pg} / \mathrm{mL}$ and 120 $\mathrm{pg} / \mathrm{mL}$, respectively. 
2 volume, ventricular volume, hippocampal volume, entorhinal cortex volume, and white

3 matter hyperintensity (WMH) volume. These volumes were normalized as fractions of

4 the intracranial volume. Cortical reconstruction and volumetric segmentation were

5 performed with the FreeSurfer image analysis suite. WMH volumes were calculated

6 based on coregistered T1-, T2-, and proton density-weighted structural MRI images.

7

8 APOE genotype

9

APOE genotyping was performed on DNA samples of each subject's blood cells

using an $A P O E$ genotyping kit. $A P O E$ includes 3 alleles $(\varepsilon 2, \varepsilon 3$, and $\varepsilon 4)$ and 6 genotypes

$11(\varepsilon 22, \varepsilon 23, \varepsilon 24, \varepsilon 33, \varepsilon 34$, and $\varepsilon 44)$. We assessed the number of $\varepsilon 4$ alleles, as the $\varepsilon 4$ allele

is known as a risk factor for $\mathrm{AD}$.

\section{HML model}

We applied HML to obtain a decision tree for MCI subtyping. HML is a type of hierarchical mixture of experts [8-10] that integrates multiple learners using a decision tree. HML divides individuals into similar groups based on various datasets of the individuals and generates appropriate predictive models for each group. As described 
1 below, HML simultaneously estimates the parameters for a decision tree and the

2 prediction models using the expectation-maximization (EM) algorithm based on

3 factorized information criterion (FIC), which is an estimator specific to HML

4 (Supplementally information). Using HML has several advantages, including the

5 following: (1) the decision tree facilitates understanding of how individuals are classified

6 into their subtypes, and (2) HML naturally prunes more complex brunches of a decision

7 tree by the contribution of FIC, providing a decision tree with higher interpretability

8 compared to them from the other methods based on decision tree. A program for HML

9 was provided by NEC Corporation.

\section{Decision tree}

A decision tree in HML was created in which the gating nodes were non-leaf nodes and the expert nodes were leaf nodes (Figure S1). The $i$-th gating node $g_{i}$ assigns an individual as input data $x^{(n)}$ to an appropriate expert node for prediction based on the rule $x\left[\gamma_{i}\right]<t_{i}$, where $\gamma_{i}$ and $t_{i}$ are the index of a variable and a threshold in a gating node $g_{i}$, respectively. A binary logistic regression model was used in the expert nodes. The prediction model in the $j$-th expert node is presented in the following equation:

$$
p\left(y^{N} \mid x^{N}, \phi_{j}\right)=\frac{1}{1+\exp \left(-\phi_{j}^{T} x^{N}\right)} .
$$


1 Let us denote the regression target as $y^{N}=y^{(1)}, \ldots, y^{(N)}$, where $y^{(n)}$ corresponds to

$2 x^{(n)}$ and $\phi_{j}$ indicates a weight vector of parameters in the $j$-th expert node.

4 Estimation of parameters by EM-like iterative optimization

To obtain a decision tree model via HML, we needed the parameters for the

6 gating nodes (i.e., $g, \gamma$, and $t$ ) and the expert nodes (i.e., $\phi)$. These parameters were

7 estimated by EM-like iterative optimization (Algorithm 1 in Supplementally

8 information). The variational distribution, which is derived from FIC, in the E-step has

9 a regularization effect and penalizes the expert nodes that contribute to the formation of complex tree structure and that have more variables with small effects (Supplementally information). Therefore, HML automatically selects an optimal decision tree and optimal model parameters to maximize FIC $[9,10]$.

Test performance

We used the datasets from $156 \mathrm{AD}$ dementia patients and $305 \mathrm{CN}$ subjects as training and validation datasets to determine a decision tree and model parameters via HML (Figure S2). The data for four-fifths of the AD dementia patients and CN subjects were used as a training dataset. The remaining data were used as a validation dataset to 
1 fine-tune the model parameters. The regression target was $y^{(n)}=1$ when a subject is

$2 \mathrm{AD}$ and $y^{(n)}=0$ when a subject is $\mathrm{CN}$. Of 480 MCI subjects, the dataset from the 396

3 MCI subjects who were followed for more than three years was used as test data. The

4 regression target was $y^{(n)}=1$ when an MCI subject converted to AD dementia within

$5 \quad$ three years and $y^{(n)}=0$ otherwise.

6 Using the training dataset, we first set the tree depth $d$ to a value ranging from

7 three to six. Then, we estimated parameters via HML. As we mentioned above, HML

8 optimizes the parameters based on the EM-like iterative optimization. It is well known

9 that the EM iterative optimization generally converges to a local optimum depending on an initial value and is not guaranteed to converge to the global optimum. To avoid a local optimum, we generated 500 models with different initial values at each depth. We next applied the validation dataset to the 2,000 models $(=4$ depths $\times 500$ models) generated from the training data and adopted the decision tree model with the highest accuracy in the validation dataset as the model with optimal parameters (Figure S2). We finally calculated the test performances of the model using the test data. These procedures were repeated for 5-fold cross-validation (CV). An HML decision tree model generated from the training data classified the MCI subjects into $\mathrm{AD}$ dementia patients (the predicted $\mathrm{ADs}$ ) or $\mathrm{CN}$ subjects (the predicted 
$1 \mathrm{CNs})$. For test performance, the predicted ADs in whom MCI converted to AD dementia

2 within three years were defined as true positives (TPS). The predicted ADs in whom MCI

3 did not convert were defined as false positives $(F P s)$. In the same way, the predicted CNs

4 who developed $\mathrm{AD}$ dementia within three years and those who did not develop $\mathrm{AD}$

5 dementia were defined as false negatives $(F N s)$ and true negatives $(T N s)$, respectively.

6 We calculated sensitivity, specificity, precision, and accuracy using the four outcomes as

7 follows:

8

9

10

$$
\begin{aligned}
\text { Sensitivity } & =\frac{T P}{F N+F P}, \\
\text { Specificity } & =\frac{T N}{F P+T N}, \\
\text { Precision } & =\frac{T P}{T P+F P}, \\
\text { Accuracy } & =\frac{T P+T N}{T P+F P+T N+F N} .
\end{aligned}
$$

The AD conversion in each MCI subject is presented as a time-to-event value, that is, the number of days from age at baseline to age at onset. In this study, we defined the data for the MCI subjects in whom MCI did not convert to AD dementia during follow-up period as censoring data. The log-rank test was performed to evaluate the difference in conversion between the predicted ADs and the predicted CNs or among the MCI subtypes. The conversion rate at time $t\left(C R_{t}\right)$ was given by the following:

$$
C R_{t}=1-\left(C R_{t-1} \frac{n_{t}-c_{t}}{n_{t}}\right)
$$


1 where $n_{t}$ is number at risk at time $t$ and $c_{t}$ is the number of the individuals converted to

$2 \mathrm{AD}$ dementia during the period from time $t$ - 1 to time $t$.

\section{Classification and regression tree (CART)}

6 training/validation/test datasets with HML (Figure S2). We set the tree depth $d$ to a value

7 ranging from three to six. A function GridSearchCV provided by the Python scikit-learn

8 package [11] optimized the following parameters in CART: the maximum depth of the

9 tree $(3,4,5$, and 6); the criterion ("the Gini impurity" or "the information gain"); the minimum number of samples required to be at a leaf node $(1, \ldots, 11)$; the minimum number of samples required to split an internal node $(2, \ldots, 11)$; the random state $(0, \ldots, 101)$; the strategy used to choose the split at each node ("best" or "random"). We adopted the model with the highest accuracy in a validation dataset and calculated the test performances of the adopted model using the test dataset.

\section{Composite scores of cognitive domains}

We used the composite scores of four cognitive domains (memory, executive

18 function, language, and visuospatial function) in a bi-factor model $[12,13]$. The 
1 composite score for memory was calculated based on the following tests: the word lists

2 for the three forms of the Alzheimer's Disease Assessment Scale-Cognitive Subscale

3 (ADAS-Cog), the word lists for the two forms of the Rey Auditory Verbal Learning Test

4 (RAVLT), the three word recall items from the MMSE, and Logical Memory scores. The

5 composite score for executive function was calculated based on the following tests: the

6 category fluency tests for animals and vegetables, the Trail-Making Test (Parts A and B),

7 the Digit Span Backwards test, the Wechsler Adult Intelligence Scale-Revised (WAIS-R)

8 Digit Symbol Substitution Test, and tests for five clock drawing items (circle, symbol,

9 numbers, hands, time). The composite score for language was calculated based on the

10 following tests: a neuropsychological battery including three language-related tests, the

11 MMSE including eight language tasks, the ADAS-Cog including three different language

12 tasks, and the Montreal Cognitive Assessment (MoCA) including six language items. The

13 composite score for visuospatial function was calculated based on the following tests: a

14 neuropsychological battery including five tests related to copying a clock, the

15 constructional praxis test in the ADAS-Cog, and the copy design test in the MMSE.

16 Detailed protocols are available for download at https://ida.loni.ucla.edu/.

baseline in the two subtypes and was applied as a multiple comparison procedure. We 
1 performed linear mixed model (LMM) analyses to compare the associations between

2 MCI subtypes and follow-up time. Subtype 2, which is mentioned in the following result

3 sections, was used as the reference. The independent variables included the subtypes,

4 follow-up time, and the interactions between subtypes and follow-up time. The covariates

5 included age, sex, and education years. The composite scores for cognitive function were

6 used as dependent variables. The random factors included the intercept and follow-up

7 time. Separate models were run for the four cognitive functions. We used the false

8 discovery rate (FDR) method to correct for multiple testing.

CSF markers for neuronal injury and the inflammatory response

We analysed the CSF levels of the neuronal injury marker Visinin-like-protein-

121 (VILIP-1), the synaptic dysfunction markers Synaptosomal-associated protein, 25kDa (SNAP-25) and Neurogranin (NGRN), and the inflammation marker YKL-40. The levels of VILIP-1, SNAP-25 and NGRN were analysed with the Erenna ${ }^{\circledR}$ immunoassay system (Singulex Inc., Alameda, CA, USA). The levels of YKL-40 were analysed with a MicroVue YKL-40 ELISA (Quidel, San Diego, CA). We analysed the marker levels at baseline in $62 \mathrm{MCI}$ subjects. 


\section{Gene expression analysis}

3 U219 Array using peripheral blood samples from ADNI subjects. Raw expression values

4 obtained directly from CEL files were pre-processed using the robust multichip average

5 (RMA) normalization method. The Affymetrix Human Genome U219 Array contains

6530,467 probes for 49,293 transcripts. All probe sets were mapped and annotated with

7 reference to the human genome (hg19). Probe sets without gene symbol names, control

8 probe sets, and non-specific probe sets were excluded from our analysis. The expression

9 levels of genes with multiple probe sets were averaged in each subject. We analysed the

10 gene expression levels in 239 MCI subjects. The gene expression levels were measured

11 in 92 subjects at baseline. We also added 147 subjects if the status at the measurement

12 visit of a gene expression was $\mathrm{MCI}$ (without conversion to $\mathrm{AD}$ dementia or reversion to

$13 \mathrm{CN}$ ) in order to analyse as many subjects as possible. ANOVA was used to test differences

14 in the gene expression levels among subtypes. Differentially expressed genes (DEGs)

15 were selected as the genes whose differences in expression had non-adjusted $\mathrm{p}$-values $<$

16 0.05. We first set the significance level of the FDR-adjusted $p$-value to $<0.05$, but we

17 found no genes at this significance level. We adopted a significance level based on a non-

18 adjusted p-value to assess the overall alterations in gene expression by gene functional 
1 enrichment analysis. Next, we classified the DEGs into clusters using agglomerative

2 hierarchical clustering based on Ward's method and the Euclidean distance. The number

3 of clusters was set to six based on the gap statistic [14]. Gene functional enrichment

4 analysis of the DEGs was performed using the Metascape database

5 (http://metascape.org/) [15].

6 We performed linear regression analysis to examine the association between CSF

7 YKL-40 levels and gene expression levels. In this analysis, we used CN subjects, AD

8 dementia patients and MCI subjects. These subjects were measured for gene expression

9 and CSF YKL-40 at the same visit. The following subjects were analysed: $23 \mathrm{CN}$ subjects,

$1018 \mathrm{MCI}$ subjects, and $19 \mathrm{AD}$ dementia patients. The independent variable was the CSF

11 YKL-40 level. The covariates included the ratio of absorbance at $260 \mathrm{~nm}$ and $280 \mathrm{~nm}$,

12 the ratio of absorbance at $260 \mathrm{~nm}$ and $230 \mathrm{~nm}$, the RNA integrity number, the microarray

13 plate number, the $A P O E \& 4$ dose, the disease status (CN, MCI, AD), age, sex, and

14 education. Gene expression levels were used as dependent variables. The genes correlated

15 with CSF YKL-40 were selected as the genes with non-adjusted p-values $<0.05$.

17 Results

18 Test performance of a decision tree model obtained by HML 
2 dementia patients and $305 \mathrm{CN}$ subjects, and assessed the test performance of the models

3 using the dataset from 396 MCI subjects by 5-fold CV (see Materials and methods,

4 Figure S2). The data for four-fifths of the $\mathrm{AD}$ dementia patients and $\mathrm{CN}$ subjects were

5 used as a training dataset. The remaining data were used as a validation dataset to fine-

6 tune the model parameters. Using the training dataset, we tried decision trees with four

7 depths ranging from three to six and generated 500 models based on the different initial

8 conditions at each depth. Next, we applied the validation dataset to the 2,000 models (=

94 depths $\times 500$ models) generated from the training data. We selected the decision tree

10 model with the highest accuracy in the validation dataset as the model with optimal

11 parameters. We further used the data for $396 \mathrm{MCI}$ subjects as test data to evaluate the

12 decision tree model and calculated the test performance of the model. These procedures

13 were repeated for 5-fold CV. The results from 5-fold CV showed a sensitivity of

$140.751 \pm 0.027$, a specificity of $0.810 \pm 0.020$, a precision of $0.682 \pm 0.022$, and an accuracy

15 of $0.789 \pm 0.014$. In the same way, we also used the CART method, which is known as a

16 traditional decision tree method, and compared the test performance. The comparison of

17 the models obtained from the two methods showed that the models from the HML had

18 higher accuracy than those from CART (Table 2). In addition, the comparison of model 
1 complexities showed that the models from HML had fewer leaf nodes (expert nodes in

2 HML) than those from the CART method, providing higher interpretability.

\section{Characteristics of each subtype}

We next focused on construction of a decision tree to examine MCI subtypes

6 (Figure 1A). This decision tree was generated from all 461 subjects, including $156 \mathrm{AD}$

7 dementia patients and $305 \mathrm{CN}$ subjects, and had five expert nodes. After the $396 \mathrm{MCI}$

8 subjects were applied, the decision tree model had an accuracy of 0.804 . We next applied

9 all $480 \mathrm{MCI}$ subjects to the decision tree. This decision tree model predicted 170 of the

10 MCI subjects as $\mathrm{AD}$ dementia patients (the predicted ADs) and 310 of the MCI subjects

11 as $\mathrm{CN}$ subjects (the predicted $\mathrm{CNs}$ ). We compared the conversion rates to $\mathrm{AD}$ dementia

12 between the predicted ADs and the predicted CNs. The predicted ADs exhibited a higher

13 percentage of progression to $\mathrm{AD}$ dementia over three years from baseline $(61.6 \%)$ than

14 the predicted CNs (11.5\%) (Figure 1B).

The individuals included in an expert node on a decision tree are a group of individuals with similar features. We then considered the MCI subjects who were classified into a specific expert node as one subtype. The MCI subjects were divided as 
1 were in subtype 4, and 37 were in subtype 5 (Table 3 ). We compared the conversion rates

2 of MCI to AD dementia in the subjects in each subtype to characterize each subtype

3 (Figures 1C and 1D). The Kaplan-Meier curves showed different conversion patterns in

4 each subtype. Notably, $67.9 \%$ of MCI cases in the subjects in subtype 5 progressed to AD

5 dementia within three years. On the other hand, the conversion rates in subtypes 1,3 , and

64 were moderate at approximately $40 \%$. Subtype 2 had a comparatively low conversion

7 rate.

8

To provide a more detailed characterization of each subtype, we compared the

9 levels of 12 variables among the subtypes (Figure 2). Subtype 2 showed high levels of

10 CSF A $\beta(1-42)$ (Figure 2A), suggesting low deposition of $A \beta$ in the brain. The levels of

11 CSF tau (CSF tTau, CSF pTau, tTau/A $\beta(1-42)$ ratio, and $\mathrm{pTau} / \mathrm{A} \beta(1-42)$ ratio), which

12 indicate the degree of $A \beta$-dependent neurofibrillary tangles, were high in subtypes 4 and

135 (Figures 2B-E). These biomarker pattern suggests that individuals classified subtypes

144 and 5 have AD pathology in the brain. Interestingly, these subtypes did not have upstream gating nodes associated with tau on the decision tree. Subtype 1 had a high

16 ventricular volume, suggesting brain atrophy (Figure 2F). This subtype also had low

17 hippocampal, whole-brain and entorhinal cortex volumes in accordance with enlargement

18 of the ventricles (Figures $2 \mathrm{G}-\mathrm{H}$ ). Low hippocampal and entorhinal cortex volumes were 
1 also observed in subtype 5 (Figures $2 \mathrm{G}$ and $2 \mathrm{I}$ ). Regarding WMH volumes, which reflect

2 white matter lesions caused by cerebral ischaemia, there were no differences among the

3 subtypes (Figure 2J), implying that most MCI subjects in this study did not present with

4 vascular dementia. Comparison of ages showed that subtypes 1 and 4 included relatively

5 older and younger MCI subjects, respectively (Figure 2K). Not surprisingly, the MCI

6 subjects in subtypes 1 and 2 did not have $A P O E \& 4$ alleles, which are genetic risk factors,

7 and all of the subjects in subtypes 3, 4, and 5 had one or two APOE \&4 alleles because the

8 decision tree had the gating nodes with $A P O E \& 4$ alleles (Figure 2L). subtypes. The spot matrix characterized the subtypes with the conversion rates shown in

Figures 1C and 1D: subtype 2, with no abnormalities had a low conversion rate; subtype

1, which had some brain atrophy, and subtypes 3 and 4, which had no abnormalities in

CSF biomarkers, had intermediate conversion rates; and subtype 5, which had both CSF biomarker abnormalities and brain atrophy, had a high conversion rate.

\section{Cognitive functions in each subtype} We compared the four composite scores for memory, executive function, 
1 score in each cognitive domain indicates high cognitive function. Comparisons among

2 subtypes showed that the scores for memory, executive function, and language of the

3 subjects in subtype 2 were basically significantly higher than those of the subjects in the

4 other subtypes (Figures 4A, 4D, and 4G). The scores for visuospatial function did not

5 show significant differences among the subtypes (Figure 4J). We next examined the

6 trajectories of these scores during the follow-up time. Figures $4 \mathrm{~B}, 4 \mathrm{E}, 4 \mathrm{H}$, and $4 \mathrm{~K}$ shows

7 the temporal changes in each subtype. Individual cognitive declines are illustrated in

8 Figure S3. We performed LMM analyses with subtype 2 (no abnormalities) as the

9 reference to compare the association between follow-up time and each score. The scores

10 of memory and executive function in subtypes $1,3,4$, and 5 declined significantly more

11 steeply than those in subtype 2 over time. Subtypes 1 and/or 4 did not show significant

12 associations for the language and visuospatial function scores. Subtype 5 consistently

13 showed the most rapid decreases in all scores. In addition, subtype 1 exhibited slower

14 declines than subtypes 3, 4, and 5, particularly for the memory and executive function

15 scores. These results show that the rate of exacerbation of cognitive decline differs

16 depending on the subtype. 
2 dysfunction, and inflammation within the brain. The CSF markers were measured in the

3 following subjects: 10 subjects in subtype 1, 18 in subtype 2, 26 in subtype 3 , and 8 in

4 subtype 5. The CSF markers were not measured in any of the subjects in subtype 4 . The

5 levels of the neuronal injury marker VILIP-1 and the synaptic dysfunction markers

6 SNAP-25 and NGRN were elevated in the subtypes in the following order: 1, 2, 3, and 5

7 (Figure 5). The levels of VILIP-1 in subtype 3 and 5 were significantly higher than that

8 in subtype 1 (Figure 5A). Additionally, the levels of SNAP-25 in subtypes 3 and 5 were

9 significantly higher than those in subtypes 1 and 2 (Figure 5B). On the other hand, the 10 level of the inflammation marker YKL-40 was the highest only in subtype 5 (Figure 5D).

11 Taken together, these results show that although subtypes 3 and 5, which exhibited CSF

12 biomarker abnormalities, displayed progression of neurological damage, the

13 inflammatory response was observed only in subtype 5, which exhibited both CSF

14 biomarker abnormalities and brain atrophy. These findings suggest that the accumulation

15 of $\mathrm{A} \beta$ and tau proteins within the brain leads to neuronal dysfunction followed by an

16 inflammatory response. Additionally, as we will mention in the Discussion, the CSF

17 markers such as VILIP1 reflect $A \beta$ - and tau-induced neuronal cell death. Therefore, these

18 markers might not have been elevated in subtype 1. 


\section{Identification of subtype-signature gene pathways}

3

4 gene expression analysis among the subtypes using peripheral blood samples from ADNI

5 subjects. Gene expression levels were measured in 49 subjects in subtype 1, 174 in

6 subtype 2, 146 in subtype 3,10 in subtype 4, and 23 in subtype 5. We found 1,080 genes

7 that were differentially expressed among subtypes by ANOVA. Agglomerative

8 hierarchical clustering classified these genes into six clusters (Figure 6A and 6B). The

9 gene expression profiles predominantly displayed dynamic alterations in gene expression in subtypes 4 and 5 . The genes in cluster $4(n=109)$ were markedly downregulated in

subtype 5. The genes in cluster 4 were significantly associated with lysosomes and autophagy (Figure 6C). Autophagy regulates lysosomal digestion to break down damaged or unnecessary organelles and proteins. Additionally, autophagy regulates excess inflammatory immune responses [16, 17], which corresponds to the increased inflammation marker levels shown in Figure 5D. The genes in cluster 6 were prominently upregulated in subtype 4 and moderately upregulated in subtype 5 and were obviously related to B cell activation (Figure 6C). The genes in cluster 3 represented were upregulated in subtype 4 and downregulated in subtype 5. The genes in cluster 3 were 
1 significantly associated with terms related to the immune response, such as

2 "Immunoglobulin production mediated immune response", in addition to RNA

3 localization (Figure 6C). These results showed that the expression levels of genes in

4 immune response pathways were drastically changed in subtypes 4 and 5 and that

5 pathways evoked by immune responses such as autophagy were also altered in subtype

6 5. In the above analysis, although we could not analyse the level of the inflammation

7 marker YKL-40 in subtype 4 because this marker was not measured in the subjects of this

8 subtype, our results suggested the occurrence of aberrant immune responses in the blood

9 of the subjects in subtype 4 similar to that occurring in the subjects in subtype 5 . However,

10 we could not directly relate the events in the blood and CSF. We then searched the genes

11 whose expression levels correlated with the levels of YKL-40 (see Materials and

12 Methods). Overall, the expression levels of 952 genes were found to be associated with

13 the levels of YKL-40. Interestingly, these genes were predominantly associated with

14 immune system process (Figures 6D and 6E). This result implies that the inflammation

15 arising in the CSF or brain propagates to the blood and triggers immune responses. These

16 associations may indirectly reflect events within the brains of the subjects.

\section{Discussion}


2 dementia within three years via the HML approach. Our decision tree model predicted

3 the MCI subjects in whom MCI converted to $\mathrm{AD}$ dementia with higher accuracy than an

4 existing decision tree algorithm (Table 2). Furthermore, the decision tree model divided

5 the MCI subjects into five subtypes based on the characteristics of that data (Figure 1A).

6 Detailed analysis showed a relationship between the speed of transition to AD for each

7 subtype and its biological characteristics.

8

The identified subtypes revealed varying conversion rates to $\mathrm{AD}$ dementia as

10 well as differing levels of CSF biomarkers and brain atrophy (Figures 1C, 1D, 2, and 3).

11 The MCI subjects were mainly categorized into three groups in terms of AD conversion:

12 those similar to $\mathrm{CN}$ subjects with low conversion rates (subtype 2); those with

13 intermediate conversion rates (subtypes 1, 3, and 4); and those similar to AD dementia

14 patients with high conversion rates (subtype 5). Furthermore, the subtypes with

15 intermediate conversion rates were separated into subtypes with CSF biomarker

16 abnormalities (subtypes 3 and 4) and a subtype with brain atrophy (subtype 1). One of

17 the differences among these subtypes was the presence or absence of $A P O E \& 4$ alleles.

$18 A P O E \& 4$ alleles have been found to lead to $\mathrm{A} \beta$ and tau accumulation in the brain [18-21], 
1 consistent with our results. As expected, the trajectory analysis of cognitive functions

2 showed that subtype 5, which had both CSF biomarker abnormalities and brain atrophy,

3 had the steepest declines over the follow-up time (Figures $4 \mathrm{~B}, 4 \mathrm{E}, 4 \mathrm{H}$, and $4 \mathrm{~K}$ ). On the

4 other hand, we observed different exacerbation rates for each score in subtypes 1, 3, and

54 , even though these subtypes had similar conversion rates. In the $\mathrm{AT}(\mathrm{N})$ system for classifying biological $\mathrm{AD}$, where $\mathrm{A}$ is amyloid, $\mathrm{T}$ is tau,

7 and $\mathrm{N}$ is neurodegeneration [22], the MCI subjects in subtype 1 were $\mathrm{A}-\mathrm{T}-\mathrm{N}+$, and the

8 subjects in subtypes 3 and 4 were $A+T+N-$. A recent study from the Alzheimer's

9 Biomarkers in Daily Practice (ABIDE) project reported that the $\mathrm{A}+\mathrm{T}+\mathrm{N}$ - classification

10 has a higher percentage of $A P O E \& 4$ carriers than the A-T-N+ classification [23].

11 Additionally, an Amsterdam cohort study has shown that cognitive functions vary among

12 different AT(N) profiles [24]. Subtypes 3, 4, and 5 are likely to develop AD because they

13 display CSF A $\beta$ and tau abnormalities defining the Alzheimer's continuum. On the other

14 hand, subtype 1 may include MCI subjects with suspected non-AD pathophysiology

15 (SNAP) that is marked by neurodegeneration without $\mathrm{A} \beta$ deposition within the brain [25].

16 A part of the MCI subjects in subtype 1 may develop dementias other than AD in the

17 future. One of the pathologies of SNAP, such as that associated with the A-T-N+

18 classification, that can be considered is limbic-predominant age-related TDP-43 
1 encephalopathy (LATE) [26]. Stratification of subjects with MCI using biomarkers for

2 other neurodegenerative diseases, including the TDP-43 protein, would provide more

3 clarity regarding the heterogeneity of MCI.

4

Subtype 1 showed relatively low levels of CSF markers for neuronal and synaptic injury (VILIP-1 and SNAP-25) despite advanced brain atrophy (Figures 5A and

7 5B). Previous studies have shown that the CSF level of VILIP-1 is associated with the

$8 \mathrm{CSF}$ A $\beta$ and p-tau levels, suggesting that VILIP-1 is a marker of neuronal degeneration

9 related to $\mathrm{A} \beta$ and tau pathologies $[27,28]$. In addition, a comparison of CSF VILIP-1 levels among $\mathrm{CN}$ subjects, MCI subjects, and AD dementia patients showed that VILIP1 levels increased year-over-year only in MCI subjects; they did not increase in the CN subjects and AD dementia patients [29]. The VILIP-1 levels in the CSF may increase during inflammation and neurodegeneration triggered by $\mathrm{A} \beta$ and tau, but they may

14 decrease after neurons have already died and brain atrophy has occurred. Based on the findings of these studies, we concluded that subtype 1 did not exhibit increases in the

16 levels of these neuronal degeneration markers because there were no prominent CSF biomarker abnormalities. Additionally, our results suggested that the MCI subjects in subtype 1 convert to the other dementia as discussed above because they did not show 
1 relatively CSF biomarker abnormalities specific to AD pathologies.

Subtypes 3 and 5 showed high levels of CSF markers for neuronal and synaptic

4 injury such as VILIP-1 and SNAP-25 (Figures 5A and 5B). The levels of these markers

5 gradually increased with the dose of $A P O E \& 4$, consistent with the findings of recent

6 studies reporting associations between these markers and APOE $\varepsilon 4$ [30, 31]. On the other

7 hand, the levels of the inflammation marker YKL-40 in the CSF were increased only in

8 subtype 5 among the subtypes except for subtype 4 (Figure 5D). Additionally, gene

9 expression analysis using blood tissues also showed that genes associated with the

inflammatory immune response were up- or downregulated specifically in subtype 4 and

5 (Figure 6C). To clarify the potential link between the CSF and the blood, we examined the genes with blood expression levels that correlated with the CSF YKL-40 level and found that they were enriched in inflammatory immune response pathways (Figures 6D and 6E). However, the direct relationship between CSF and blood is unclear. The bloodbrain barrier (BBB) and the blood-CSF barrier play roles as boundaries between the blood and the brain or CSF. The BBB strictly regulates the passage of select blood molecules through various channels into the brain. It has been considered that the central nervous system, including the brain, has immune privilege (which protects it from inflammation 
1 and the immune response) because of the existence of the BBB. However, recent studies

2 have shown that the immune privilege of the brain is not always assured $[32,33]$. In

3 neurodegenerative diseases, including $\mathrm{AD}$, the $\mathrm{BBB}$ is impaired, exhibiting increased

4 permeability $[34,35]$. The increased permeability of the BBB enables transmission of

5 peripheral inflammation to the brain $[34,36]$. In addition, inflammatory cytokines and

6 chemokines released from activated microglia and astrocytes can stimulate peripheral

7 immune cells through a disrupted BBB [37]. This evidence suggests that the events in the

$8 \mathrm{CSF}$ and brain propagate to the blood via the disrupted BBB, and vice versa. Furthermore,

9 it is well known that inflammatory mediators enhance autophagic activity [38, 39], which

10 corresponds with the alterations in gene expression in the autophagy pathway in subtype

11 5. In summary, BBB impairment may progress in MCI subjects in subtypes 5. Our study has several limitations. First, we were not able to analyse all MCI patients in some analyses. For example, the MCI subjects in subtype 4 did not have the CSF markers for neuronal, synaptic injury, and inflammation markers. Second, although we examined the comprehensive mRNA levels in the blood, the levels of mRNA in the blood may change depending on lifestyle and other factors. To verify our results, detailed analysis using animal models is necessary. 


\section{Conclusion}

In this study, we demonstrated that HML is useful for the classification of MCI

4 subjects. Our study found some subtypes with characteristics similar to those of typical

$5 \mathrm{AD}$ and identified subtypes in whom MCI was likely to convert to other

6 neurodegenerative diseases. These findings imply that inclusion of additional

7 pathological information can enable more precise prediction of the onset or progression

8 of a wide variety of neurodegenerative diseases. Moreover, we developed a decision tree

9 model to predict conversion to AD dementia. Although there is room for improvement in

10 the overall performance of the model, focusing on specific subtypes in which conversion

11 to $\mathrm{AD}$ dementia can be predicted with the most accuracy (e.g., subtype 5, in which the

12 prediction was made with high precision) and targeting of individuals with MCI that are

13 classified as $\mathrm{AD}$ dementia patients by the prediction model for that subtype (i.e., the

14 predicted ADs) could enable more efficient clinical trials to be conducted.

16 Abbreviations

Mild cognitive impairment (MCI); Alzheimer's disease (AD); Cerebrospinal

18 fluid (CSF); Heterogeneous mixture learning (HML); Apolipoprotein E (APOE); 
1 Amyloid-beta (A $\beta)$; Expectation-maximization (EM) algorithm; Alzheimer's Disease

2 Neuroimaging Initiative (ADNI); Magnetic resonance imaging (MRI); Positron emission

3 tomography (PET); Cognitively normal (CN); Mini-Mental State Examination (MMSE);

4 Clinical Dementia Rating-Sum of Boxes (CDR-SB); Total tau (tTau); Phosphorylated tau

5 (pTau); White matter hyperintensity (WMH); Alzheimer's Disease Assessment Scale-

6 Cognitive Subscale (ADAS-Cog); Rey Auditory Verbal Learning Test (RAVLT);

7 Wechsler Adult Intelligence Scale-Revised (WAIS-R); Montreal Cognitive Assessment

8 (MoCA); Linear mixed model (LMM); False discovery rate (FDR); Visinin-like-protein-

91 (VILIP-1); Synaptosomal-associated protein, 25kDa (SNAP-25); Neurogranin

10 (NGRN); Differentially expressed genes (DEGs); Cross-validation (CV); Classification

11 and regression tree (CART); Blood-brain barrier (BBB)

Acknowledgements

We thank all the participants and staff of ADNI.

Authors' contributions 
1 computational analysis: M.K.; paper writing: M.K.; paper review and supervision:

2 K.Kasuga, A.M., T.I., E.Y., Y.F., T.T., S.M., K.Kamijo, and Y.M.. The authors read and

3 approved the final manuscript.

4

$5 \quad$ Funding

6 This work was supported by a Grant-in-Aid for Scientific Research (grant number

720 K15778 to M.K.) from the Ministry of Education, Culture, Sports, Science and

8 Technology (MEXT); the Japan Agency for Medical Research and Development

9 (AMED) (grant number JP20dk0207045 to M.K. and T.I., JP20ek0109392 to M.K., and

10 JP20dm0207073 to T.I.). The funders had no role in the study design, data collection,

11 decision to publish, or preparation of the manuscript.

Availability of data and materials

The data used during this study are available from the ADNI database

(https://ida.loni.ucla.edu/).

Ethics approval and consent to participate

This study was approved by the Ethics Committee of Osaka University. 


\section{Consent for publication}

3 Consent for publication has been granted by ADNI administrators.

4

\section{Conflicts of interest}

7 with sponsorship by the NEC Corporation. K.Kobayashi., E.Y., Y.F., and K.Kamijo. are

8 employees of NEC Corporation. The funder (NEC Corporation) provided support to

9 authors in the form of salaries (K.Kobayashi., E.Y., Y.F., and K.Kamijo.) but did not have any additional roles in the study design, data collection, decision to publish. All other

11 authors declare no competing interests.

\section{References}

14 1. World Alzheimer Report 2015: The Global Impact of Dementia.

\section{5 https://www.alz.co.uk/research/world-report-2015.}

16 2. Cummings J, Lee G, Ritter A, Sabbagh M, Zhong K. Alzheimer's disease drug

17 development pipeline: 2019. Alzheimers Dement (N Y). 2019;5:272-93.

18 3. Petersen RC, Morris JC. Mild cognitive impairment as a clinical entity and 
1 treatment target. Arch Neurol. 2005;62(7):1160-3; discussion 7.

2 4. Farias ST, Mungas D, Reed BR, Harvey D, DeCarli C. Progression of mild 3 cognitive impairment to dementia in clinic- vs community-based cohorts. Arch Neurol.

$4 \quad 2009 ; 66(9): 1151-7$.

5 5. Machulda MM, Lundt ES, Albertson SM, Kremers WK, Mielke MM, Knopman

6 DS, et al. Neuropsychological subtypes of incident mild cognitive impairment in the

$7 \quad$ Mayo Clinic Study of Aging. Alzheimers Dement. 2019;15(7):878-87.

8 6. Blanken AE, Jang JY, Ho JK, Edmonds EC, Han SD, Bangen KJ, et al. Distilling

9 Heterogeneity of Mild Cognitive Impairment in the National Alzheimer Coordinating

10 Center Database Using Latent Profile Analysis. JAMA Netw Open. 2020;3(3):e200413.

11 Mueller SG, Weiner MW, Thal LJ, Petersen RC, Jack C, Jagust W, et al. The

12 Alzheimer's disease neuroimaging initiative. Neuroimaging Clin N Am. 2005;15(4):869-

1377, xi-xii.

14 8. Jordan MI, Jacobs RA. Hierarchical Mixtures of Experts and the Em Algorithm.

15 Neural Comput. 1994;6(2):181-214.

169 Fujimaki R, Morinaga S. Factorized Asymptotic Bayesian Inference for Mixture

17 Modeling. In: Neil DL, Mark G, editors. Proceedings of the Fifteenth International

18 Conference on Artificial Intelligence and Statistics; Proceedings of Machine Learning 
1 Research: PMLR; 2012.p. 400--8.

2 10. Eto R, Fujimaki R, Morinaga S, Tamano H. Fully-Automatic Bayesian Piecewise

3 Sparse Linear Models. Jmlr Worksh Conf Pro. 2014;33:238-46.

4 11. Pedregosa F, Varoquaux G, Gramfort A, Michel V, Thirion B, Grisel O, et al.

5 Scikit-learn: Machine Learning in Python. J Mach Learn Res. 2011;12:2825-30.

6 12. Gibbons LE, Carle AC, Mackin RS, Harvey D, Mukherjee S, Insel P, et al. A 7 composite score for executive functioning, validated in Alzheimer's Disease

8 Neuroimaging Initiative (ADNI) participants with baseline mild cognitive impairment.

$9 \quad$ Brain Imaging Behav. 2012;6(4):517-27.

10 13. Crane PK, Carle A, Gibbons LE, Insel P, Mackin RS, Gross A, et al.

11 Development and assessment of a composite score for memory in the Alzheimer's Disease

12 Neuroimaging Initiative (ADNI). Brain Imaging Behav. 2012;6(4):502-16.

13 14. Tibshirani R, Walther G, Hastie T. Estimating the number of clusters in a data 14 set via the gap statistic. J Roy Stat Soc B. 2001;63:411-23.

15 15. Zhou Y, Zhou B, Pache L, Chang M, Khodabakhshi AH, Tanaseichuk O, et al. 16 Metascape provides a biologist-oriented resource for the analysis of systems-level 17 datasets. Nat Commun. 2019;10(1):1523.

18 16. Giegerich AK, Kuchler L, Sha LK, Knape T, Heide H, Wittig I, et al. Autophagy- 
1 dependent PELI3 degradation inhibits proinflammatory IL1B expression. Autophagy.

$2 \quad 2014 ; 10(11): 1937-52$.

3 17. Lee JP, Foote A, Fan H, Peral de Castro C, Lang T, Jones SA, et al. Loss of

4 autophagy enhances MIF/macrophage migration inhibitory factor release by

5 macrophages. Autophagy. 2016;12(6):907-16.

6 18. Bi X, Yong AP, Zhou J, Ribak CE, Lynch G. Rapid induction of intraneuronal

7 neurofibrillary tangles in apolipoprotein E-deficient mice. Proc Natl Acad Sci U S A.

$8 \quad 2001 ; 98(15): 8832-7$.

9 19. DeMattos RB, Cirrito JR, Parsadanian M, May PC, O'Dell MA, Taylor JW, et al.

10 ApoE and clusterin cooperatively suppress Abeta levels and deposition: evidence that

11 ApoE regulates extracellular Abeta metabolism in vivo. Neuron. 2004;41(2):193-202.

12 20. Tiraboschi P, Hansen LA, Masliah E, Alford M, Thal LJ, Corey-Bloom J. Impact

13 of APOE genotype on neuropathologic and neurochemical markers of Alzheimer disease.

14 Neurology. 2004;62(11):1977-83.

15 21. Small SA, Duff K. Linking Abeta and tau in late-onset Alzheimer's disease: a

16 dual pathway hypothesis. Neuron. 2008;60(4):534-42.

17 22. Jack CR, Jr., Bennett DA, Blennow K, Carrillo MC, Feldman HH, Frisoni GB,

18 et al. A/T/N: An unbiased descriptive classification scheme for Alzheimer disease 
1 biomarkers. Neurology. 2016;87(5):539-47.

2 23. Altomare D, de Wilde A, Ossenkoppele R, Pelkmans W, Bouwman F, Groot C,

3 et al. Applying the ATN scheme in a memory clinic population: The ABIDE project.

$4 \quad$ Neurology. 2019;93(17):e1635-e46.

5 24. Ebenau JL, Timmers T, Wesselman LMP, Verberk IMW, Verfaillie SCJ, Slot

6 RER, et al. ATN classification and clinical progression in subjective cognitive decline:

7 The SCIENCe project. Neurology. 2020;95(1):e46-e58.

8 25. Jack CR, Jr., Knopman DS, Weigand SD, Wiste HJ, Vemuri P, Lowe V, et al. An

9 operational approach to National Institute on Aging-Alzheimer's Association criteria for 10 preclinical Alzheimer disease. Ann Neurol. 2012;71(6):765-75.

11 26. Nelson PT, Dickson DW, Trojanowski JQ, Jack CR, Boyle PA, Arfanakis K, et 12 al. Limbic-predominant age-related TDP-43 encephalopathy (LATE): consensus working 13 group report. Brain. 2019;142(6):1503-27.

14 27. Sutphen CL, McCue L, Herries EM, Xiong C, Ladenson JH, Holtzman DM, et 15 al. Longitudinal decreases in multiple cerebrospinal fluid biomarkers of neuronal injury 16 in symptomatic late onset Alzheimer's disease. Alzheimers Dement. 2018;14(7):869-79.

17 28. Zhang H, Ng KP, Therriault J, Kang MS, Pascoal TA, Rosa-Neto P, et al. 18 Cerebrospinal fluid phosphorylated tau, visinin-like protein-1, and chitinase-3-like 
1 protein 1 in mild cognitive impairment and Alzheimer's disease. Transl Neurodegener.

$2 \quad 2018 ; 7: 23$.

3 29. Kester MI, Teunissen CE, Sutphen C, Herries EM, Ladenson JH, Xiong C, et al.

4 Cerebrospinal fluid VILIP-1 and YKL-40, candidate biomarkers to diagnose, predict and

5 monitor Alzheimer's disease in a memory clinic cohort. Alzheimers Res Ther.

$6 \quad 2015 ; 7(1): 59$.

7 30. Wang S, Zhang J, Pan T. APOE $\varepsilon 4$ is associated with higher levels of CSF SNAP-

825 in prodromal Alzheimer's disease. Neurosci Lett. 2018;685:109-13.

9 31. Wang L, Zhang M, Wang Q, Jiang X, Li K, Liu J. APOE $\varepsilon 4$ Allele Is Associated 10 with Elevated Levels of CSF VILIP-1 in Preclinical Alzheimer's Disease. Neuropsychiatr

11 Dis Treat. 2020;16:923-31.

12 32. McManus RM, Heneka MT. Role of neuroinflammation in neurodegeneration:

13 new insights. Alzheimers Res Ther. 2017;9(1):14.

14 33. Forrester JV, McMenamin PG, Dando SJ. CNS infection and immune privilege.

15 Nat Rev Neurosci. 2018;19(11):655-71.

16 34. Takeda S, Sato N, Ikimura K, Nishino H, Rakugi H, Morishita R. Increased

17 blood-brain barrier vulnerability to systemic inflammation in an Alzheimer disease mouse

18 model. Neurobiol Aging. 2013;34(8):2064-70. 
$135 . \quad$ Nelson AR, Sweeney MD, Sagare AP, Zlokovic BV. Neurovascular dysfunction

2 and neurodegeneration in dementia and Alzheimer's disease. Biochim Biophys Acta.

$3 \quad 2016 ; 1862(5): 887-900$.

4 36. Hoogland IC, Houbolt C, van Westerloo DJ, van Gool WA, van de Beek D.

5 Systemic inflammation and microglial activation: systematic review of animal

6 experiments. J Neuroinflammation. 2015;12:114.

7 37. Goldeck D, Witkowski JM, Fülop T, Pawelec G. Peripheral Immune Signatures

8 in Alzheimer Disease. Curr Alzheimer Res. 2016;13(7):739-49.

9 38. Deretic V, Saitoh T, Akira S. Autophagy in infection, inflammation and immunity.

10 Nat Rev Immunol. 2013;13(10):722-37.

11 39. Uddin MS, Stachowiak A, Mamun AA, Tzvetkov NT, Takeda S, Atanasov AG, 12 et al. Autophagy and Alzheimer's Disease: From Molecular Mechanisms to Therapeutic 13 Implications. Front Aging Neurosci. 2018;10:04.

Figure legends

Figure 1. Characterization of MCI subtypes.

18 (A) Decision tree model for prediction of conversion to $\mathrm{AD}$ dementia. The dotted lines 
1 represent each threshold. $(\mathrm{B}, \mathrm{C})$ Conversion rates over time in the predicted ADs and CNs

$2 \quad(p=3.15 \mathrm{e}-35$ in the log-rank test $)(B)$ and in each subtype $(p=4.62 \mathrm{e}-15$ in the log-rank

3 test) (C). (D) Conversion rates within three years in each MCI subtype.

4

5 Figure 2. Features in each subtype.

6 (A) CSF A $\beta(1-42)$ level, (B) CSF tTau level, (C) CSF pTau level, (D) the tTau/A $\beta(1-42)$

7 ratio, (E) the pTau/A $(1-42)$ ratio, (F) the normalized ventricular volume, (G) the

8 normalized hippocampal volume, $(\mathrm{H})$ the normalized whole-brain volume, (I) the

9 normalized entorhinal cortex volume, $(\mathrm{J})$ the normalized $\mathrm{WMH}$ volume, $(\mathrm{K})$ age, and (L)

$10 A P O E \& 4$ alleles. The blue and red lines indicate the cut-off values based on the $\mathrm{CN}$

11 subjects and the $\mathrm{AD}$ dementia patients. Points below the blue line or above the red line

12 represent levels similar to those in $\mathrm{AD}$ dementia patients.

Figure 3. Spot matrix showing the proportions of individuals with features similar

to those of $\mathrm{AD}$ dementia patients.

16 The spot size represents the proportion of individuals with values exceeding the cut-off

17 value. Yellow indicates a value greater than $50 \%$. 
1 Figure 4. Cognitive functions in each subtype.

2 (A, D, G, J) Comparisons of cognitive functions at baseline among subtypes. The Tukey’s

3 HSD test was used to verify the differences in scores between two subtypes and was

4 applied as a multiple comparison procedure. (B, E, H, K) Longitudinal changes in

5 cognitive functions by subtype. Each trajectory indicates the linear regression lines. The

6 error bars represent the $95 \%$ confidence intervals. (C, F, I, L) Temporal cognitive decline

7 compared with subtype2. The bar plots represent the interactions between subtypes and

8 follow-up time, as estimated by the LMM with subtype 2 as a reference. ${ }^{*} \mathrm{p}<0.05, * * \mathrm{p}$

$9<0.01, * * * \mathrm{p}<0.001$

Figure 5. Levels of the CSF neuronal and synaptic injury markers VILIP-1 (A),

SNAP-25 (B), and NGRN (C) and the inflammatory response marker YKL-40 (D).

The Tukey's HSD test was used to verify the differences in CSF levels between two subtypes and was applied as a multiple comparison procedure. ${ }^{*} \mathrm{p}<0.05,{ }^{* *} \mathrm{p}<0.01$, $* * * \mathrm{p}<0.001$.

Figure 6. Gene expression analysis using peripheral blood samples.

(A) Heatmap and hierarchical clustering of 1,080 DEGs. The colour represents the 
1 normalized gene expression level (z-score). Each row represents each gene. Clustering

2 was performed via Ward's method. The number of clusters was set to six based on the gap

3 statistic (Figure S4). (B) Gene expression profiles of the six clusters. The black and red

4 polygonal lines represent the $\mathrm{z}$-scores of the genes and the averaged $\mathrm{z}$-scores, respectively.

5 (C) Gene functional enrichment analysis of the genes in each cluster. Each bar shows the

6 significances of the enriched terms for the genes in each cluster. (D, E) Parent terms (D)

7 and immune system pathways (E) of the genes that were correlated with the inflammation

8 marker YKL-40. The bars in (E) indicate the enrichment of terms with the parent term

$9 \quad$ "Immune system process".

10

\begin{tabular}{|c|c|c|c|}
\hline & $\mathrm{CN}$ & $\mathrm{MCl}$ & $A D$ \\
\hline $\mathrm{N}$ & 305 & 480 & 156 \\
\hline Age in years, mean $\pm s . d$ & $73.7 \pm 5.73$ & $71.8 \pm 7.42$ & $74 \pm 8.32$ \\
\hline Sex (Female:Male) & $162: 143$ & $200: 280$ & 69:87 \\
\hline Education year, mean \pm s.d. & $16.3 \pm 2.64$ & $16.0 \pm 2.78$ & $15.5 \pm 2.67$ \\
\hline CSF A $\beta(1-42)(p g / m L)$, mean \pm s.d. & $1226.0 \pm 441.4$ & $964.2 \pm 436.8$ & $644.6 \pm 286.9$ \\
\hline CSF tTau (pg/mL), mean \pm s.d. & $238.4 \pm 89.6$ & $287.4 \pm 137.2$ & $373.8 \pm 138.6$ \\
\hline CSF pTau $(\mathrm{pg} / \mathrm{mL})$, mean \pm s.d. & $21.9 \pm 9.24$ & $27.9 \pm 15.2$ & $37.3 \pm 14.7$ \\
\hline tTau / A $(1-42)$, mean \pm s.d. & $2.33 \mathrm{e}-01 \pm 1.57 \mathrm{e}-01$ & $3.86 \mathrm{e}-01 \pm 2.90 \mathrm{e}-01$ & $6.51 e-01 \pm 3.07 e-01$ \\
\hline pTau / A $\beta(1-42)$, mean \pm s.d. & $2.19 \mathrm{e}-02 \pm 1.71 \mathrm{e}-02$ & $3.82 \mathrm{e}-02 \pm 3.13 \mathrm{e}-02$ & $6.52 \mathrm{e}-02 \pm 3.18 \mathrm{e}-02$ \\
\hline Whole brain volume / ICV, mean \pm s.d. & $6.94 \mathrm{e}-01 \pm 4.40 \mathrm{e}-02$ & $6.83 e-01 \pm 5.00 e-02$ & $6.45 \mathrm{e}-01 \pm 4.21 \mathrm{e}-02$ \\
\hline Hippocampus volume / ICV, mean \pm s.d. & $5.01 e-03 \pm 5.88 \mathrm{e}-04$ & $4.50 \mathrm{e}-03 \pm 8.19 \mathrm{e}-04$ & $3.84 e-03 \pm 6.58 \mathrm{e}-04$ \\
\hline Ventricle volume / ICV, mean \pm s.d. & $2.14 \mathrm{e}-02 \pm 1.03 \mathrm{e}-02$ & $2.45 \mathrm{e}-02 \pm 1.29 \mathrm{e}-02$ & $3.05 \mathrm{e}-02 \pm 1.16 \mathrm{e}-02$ \\
\hline Entorhinal cortex volume / ICV, mean \pm s.d. & $2.58 \mathrm{e}-03 \pm 3.77 \mathrm{e}-04$ & $2.33 \mathrm{e}-03 \pm 4.79 \mathrm{e}-04$ & $1.86 \mathrm{e}-03 \pm 4.35 \mathrm{e}-04$ \\
\hline WMH volume, mean \pm s.d. & $4.03 \pm 7.49$ & $5.02 \pm 8.44$ & $4.75 \pm 6.73$ \\
\hline APOE $\varepsilon 4$ carriers (\%) & $81(26.6 \%)$ & $239(49.8 \%)$ & $108(69.2 \%)$ \\
\hline
\end{tabular}


Table2. Test performances of HML and CART

\begin{tabular}{|c|c|c}
\hline & HML & CART \\
\hline Sensitivity & $0.751 \pm 0.027$ & $0.695 \pm 0.054$ \\
\hline Specificity & $0.810 \pm 0.020$ & $0.787 \pm 0.023$ \\
\hline Precision & $0.682 \pm 0.022$ & $0.638 \pm 0.016$ \\
\hline Accuracy & $0.789 \pm 0.014$ & $0.755 \pm 0.013$ \\
\hline \# leaf nodes & $3.8 \pm 1.095$ & $19 \pm 4.301$ \\
\hline
\end{tabular}

2

Table 3. The summary of each subtype.

\begin{tabular}{|c|c|c|c|c|c|}
\hline & Subtype 1 & Subtype 2 & Subtype 3 & Subtype 4 & Subtype 5 \\
\hline $\mathrm{N}$ (subjects with follow-up $\geq 3$ years) & $68(57)$ & $173(143)$ & $188(155)$ & $14(13)$ & $37(28)$ \\
\hline Age in years, mean \pm s.d. & $73.9 \pm 8.16$ & $71.8 \pm 7.47$ & $71.4 \pm 7.25$ & $66 \pm 6.07$ & $72 \pm 5.76$ \\
\hline Sex (Female:Male) & $25: 43$ & 72:101 & $82: 106$ & 9:5 & $12: 25$ \\
\hline Education year, mean \pm s.d. & $15.8 \pm 2.93$ & $16.3 \pm 2.66$ & $15.8 \pm 2.82$ & $17 \pm 2.83$ & $16.1 \pm 2.75$ \\
\hline $\operatorname{CSF} A \beta(1-42)(p g / m L)$, mean \pm s.d. & $923.3 \pm 382.3$ & $1211 \pm 425.1$ & $852.1 \pm 388.3$ & $713.9 \pm 254.5$ & $549.7 \pm 184.9$ \\
\hline CSF tTau $(\mathrm{pg} / \mathrm{mL})$, mean \pm s.d. & $273.2 \pm 145.3$ & $235.8 \pm 98.54$ & $323.7 \pm 144.6$ & $416 \pm 212.6$ & $321.7 \pm 111.2$ \\
\hline CSF pTau $(\mathrm{pg} / \mathrm{mL})$, mean \pm s.d. & $25.9 \pm 16.1$ & $22.1 \pm 11.1$ & $32 \pm 15.7$ & $42.6 \pm 24.3$ & $31.6 \pm 11.9$ \\
\hline tTau / $A \beta(1-42)$, mean \pm s.d. & $3.53 \mathrm{e}-01 \pm 2.35 \mathrm{e}-01$ & $2.37 \mathrm{e}-01 \pm 1.79 \mathrm{e}-01$ & $4.66 \mathrm{e}-01 \pm 3.01 \mathrm{e}-01$ & $6.95 \mathrm{e}-01 \pm 5.51 \mathrm{e}-01$ & $6.25 \mathrm{e}-01 \pm 2.16 \mathrm{e}-01$ \\
\hline pTau / A $\beta(1-42)$, mean \pm s.d. & $3.4 \mathrm{e}-02 \pm 2.53 \mathrm{e}-02$ & $2.29 \mathrm{e}-02 \pm 2 \mathrm{e}-02$ & $4.68 \mathrm{e}-02 \pm 3.26 \mathrm{e}-02$ & $7.21 \mathrm{e}-02 \pm 6.21 \mathrm{e}-02$ & $6.13 \mathrm{e}-02 \pm 2.18 \mathrm{e}-02$ \\
\hline Whole brain volume / ICV, mean \pm s.d. & $6.49 \mathrm{e}-01 \pm 4.02 \mathrm{e}-02$ & $6.99 \mathrm{e}-01 \pm 4.77 \mathrm{e}-02$ & $6.81 \mathrm{e}-01 \pm 5 \mathrm{e}-02$ & $7.24 \mathrm{e}-01 \pm 3.13 \mathrm{e}-02$ & $6.72 \mathrm{e}-01 \pm 4.18 \mathrm{e}-02$ \\
\hline Hippocampus volume / ICV, mean \pm s.d. & $3.83 e-03 \pm 6.33 e-04$ & $4.87 \mathrm{e}-03 \pm 6.81 \mathrm{e}-04$ & $4.49 \mathrm{e}-03 \pm 8.23 \mathrm{e}-04$ & $4.91 \mathrm{e}-03 \pm 8.1 \mathrm{e}-04$ & $3.87 \mathrm{e}-03 \pm 5.1 \mathrm{e}-04$ \\
\hline Ventricle volume / ICV, mean \pm s.d. & $3.26 \mathrm{e}-02 \pm 1.39 \mathrm{e}-02$ & $2.21 \mathrm{e}-02 \pm 1.11 \mathrm{e}-02$ & $2.44 \mathrm{e}-02 \pm 1.36 \mathrm{e}-02$ & $1.18 \mathrm{e}-02 \pm 2.49 \mathrm{e}-03$ & $2.61 \mathrm{e}-02 \pm 8.25 \mathrm{e}-03$ \\
\hline Entorhinal cortex volume / ICV, mean \pm s.d. & $1.83 e-03 \pm 2.85 e-04$ & $2.59 \mathrm{e}-03 \pm 2.95 \mathrm{e}-04$ & $2.3 e-03 \pm 5.01 e-04$ & $2.63 e-03 \pm 5.32 e-04$ & $2.05 \mathrm{e}-03 \pm 4.01 \mathrm{e}-04$ \\
\hline WMH volume, mean \pm s.d. & $5.78 \pm 9.68$ & $5.48 \pm 8.2$ & $4.29 \pm 8.47$ & $4.71 \pm 6.38$ & $5.22 \pm 7.63$ \\
\hline APOE $\varepsilon 4$ carriers (\%) & $0(0 \%)$ & $0(0 \%)$ & $188(100 \%)$ & $14(100 \%)$ & $37(100 \%)$ \\
\hline
\end{tabular}




\section{Figures}

\section{Figure 3}

The proportion of individuals with values exceeding the cut-off value
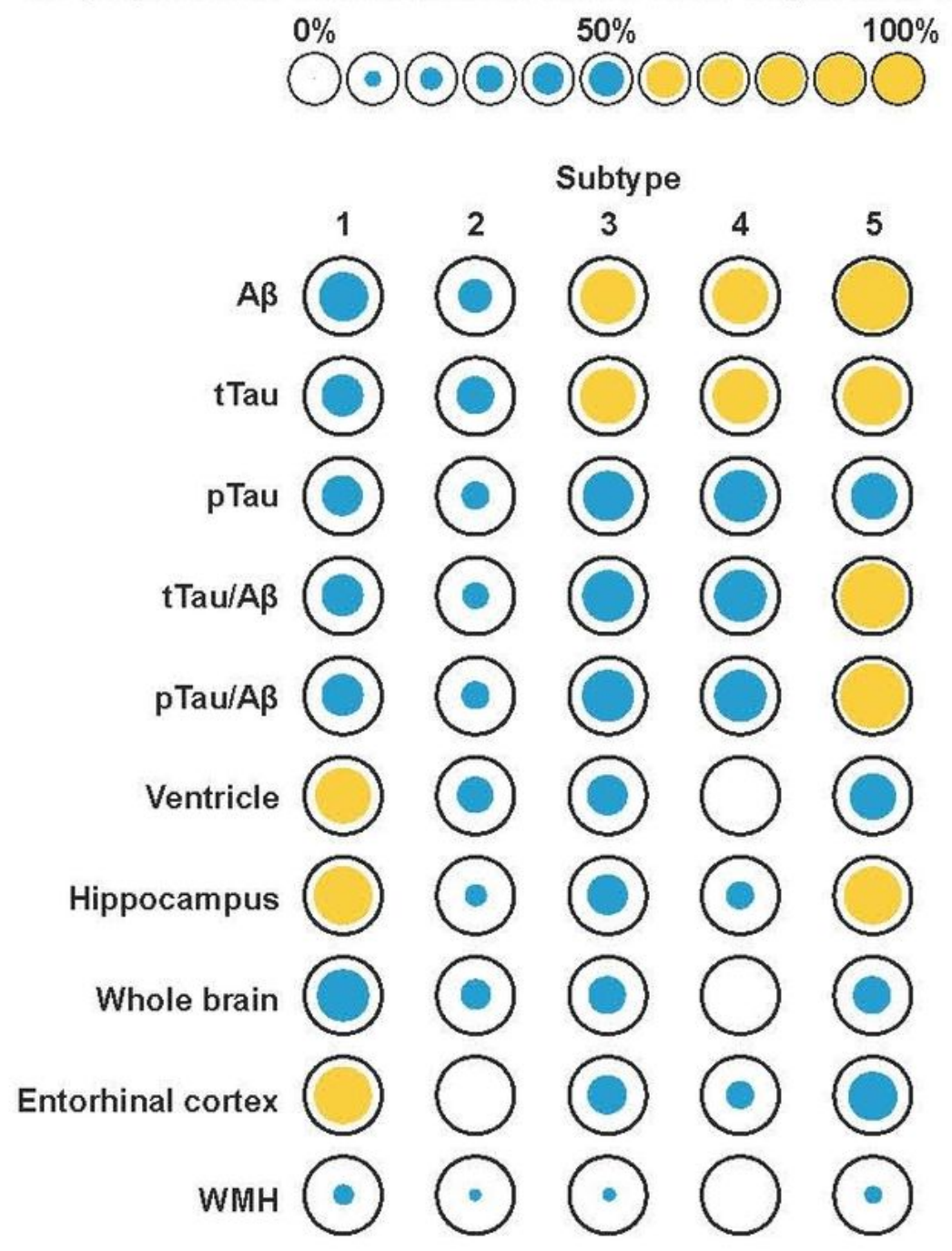

\section{Figure 1}

Spot matrix showing the proportions of individuals with features similar to those of AD dementia patients. The spot size represents the proportion of individuals with values exceeding the cut-off value. Yellow indicates a value greater than $50 \%$. 


\section{Figure 4}
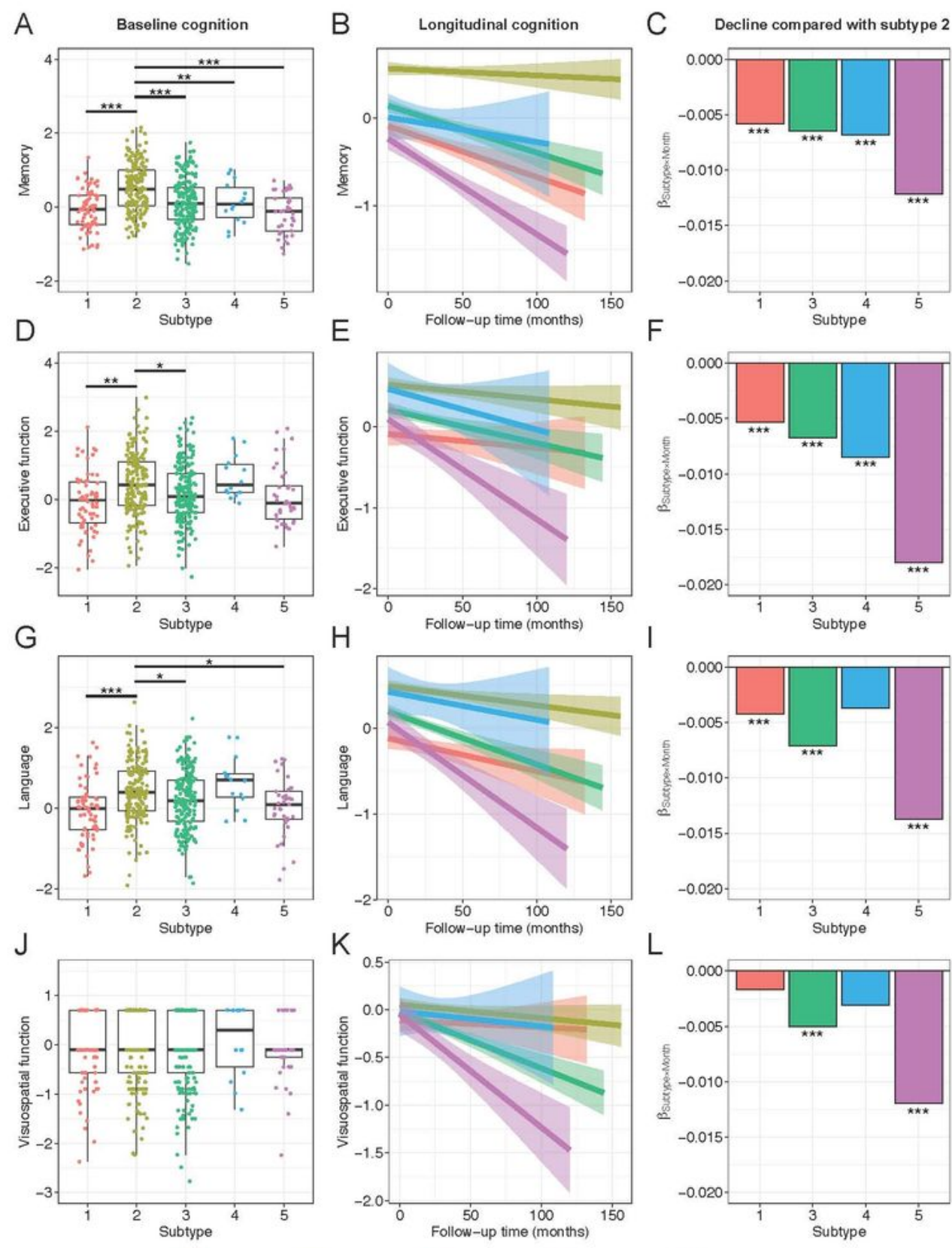

$\square$ Subtype1 $\square$ Subtype2 $\square$ Subtype3 $\square$ Subtype4 $\square$ Subtype5

\section{Figure 1}

Cognitive functions in each subtype. (A, D, G, J) Comparisons of cognitive functions at baseline among subtypes. The Tukey's HSD test was used to verify the differences in scores between two subtypes and was applied as a multiple comparison procedure. $(B, E, H, K)$ Longitudinal changes in cognitive functions by subtype. Each trajectory indicates the linear regression lines. The error bars represent the $95 \%$ confidence intervals. (C, F, I, L) Temporal cognitive decline compared with subtype2. The bar plots 
represent the interactions between subtypes and follow-up time, as estimated by the LMM with subtype 2 as a reference. ${ }^{*} p<0.05,{ }^{* \star} p<0.01,{ }^{* \star *} p<0.001$.

\section{Figure 1}

A

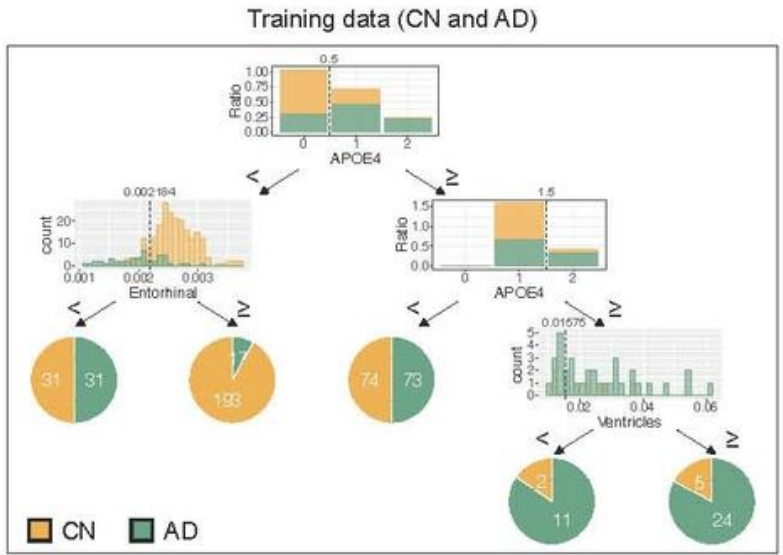

Applying to $\mathrm{MCl}$ subjects

$\mathrm{MCl}$ subtypes

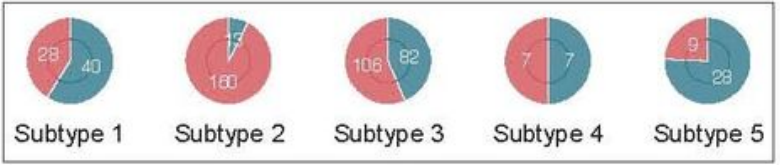

$\square$ Predicted CN $\square$ Predicted AD

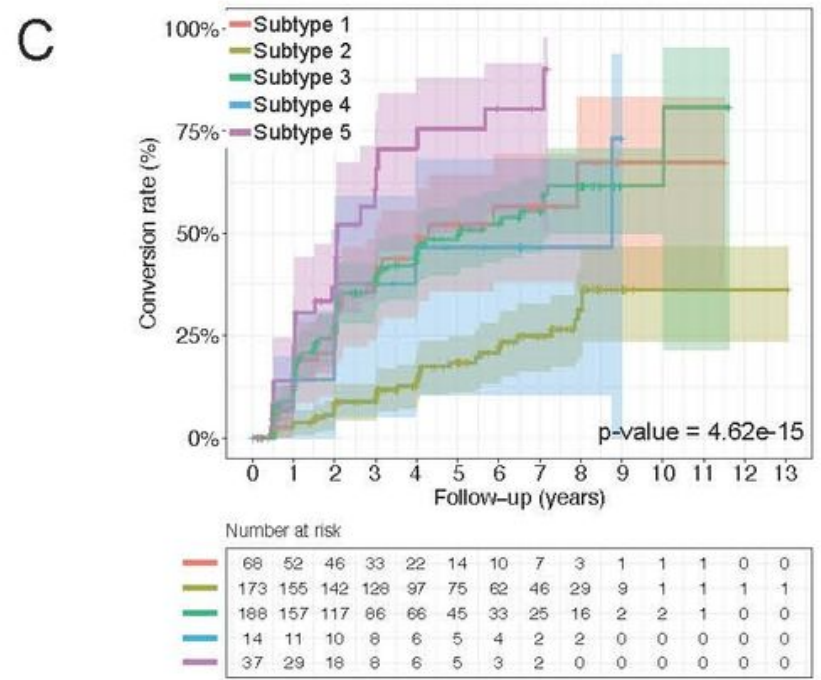

B
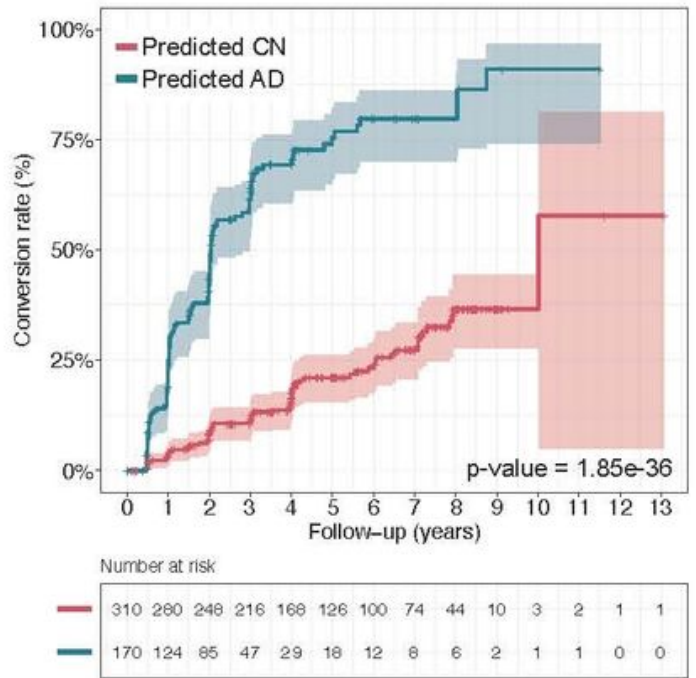

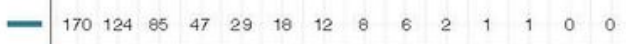

D

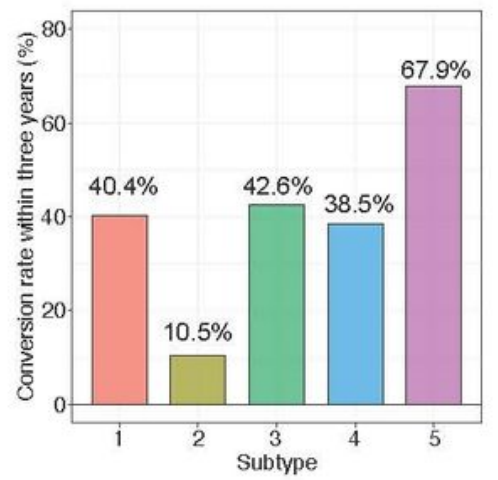

\section{Figure 1}

Characterization of $\mathrm{MCl}$ subtypes. (A) Decision tree model for prediction of conversion to AD dementia. The dotted lines represent each threshold. $(B, C)$ Conversion rates over time in the predicted ADs and CNs 
( $p=3.15 e-35$ in the log-rank test) (B) and in each subtype ( $p=4.62 e-15$ in the log-rank test) (C). (D) Conversion rates within three years in each $\mathrm{MCl}$ subtype.

\section{Figure 2}
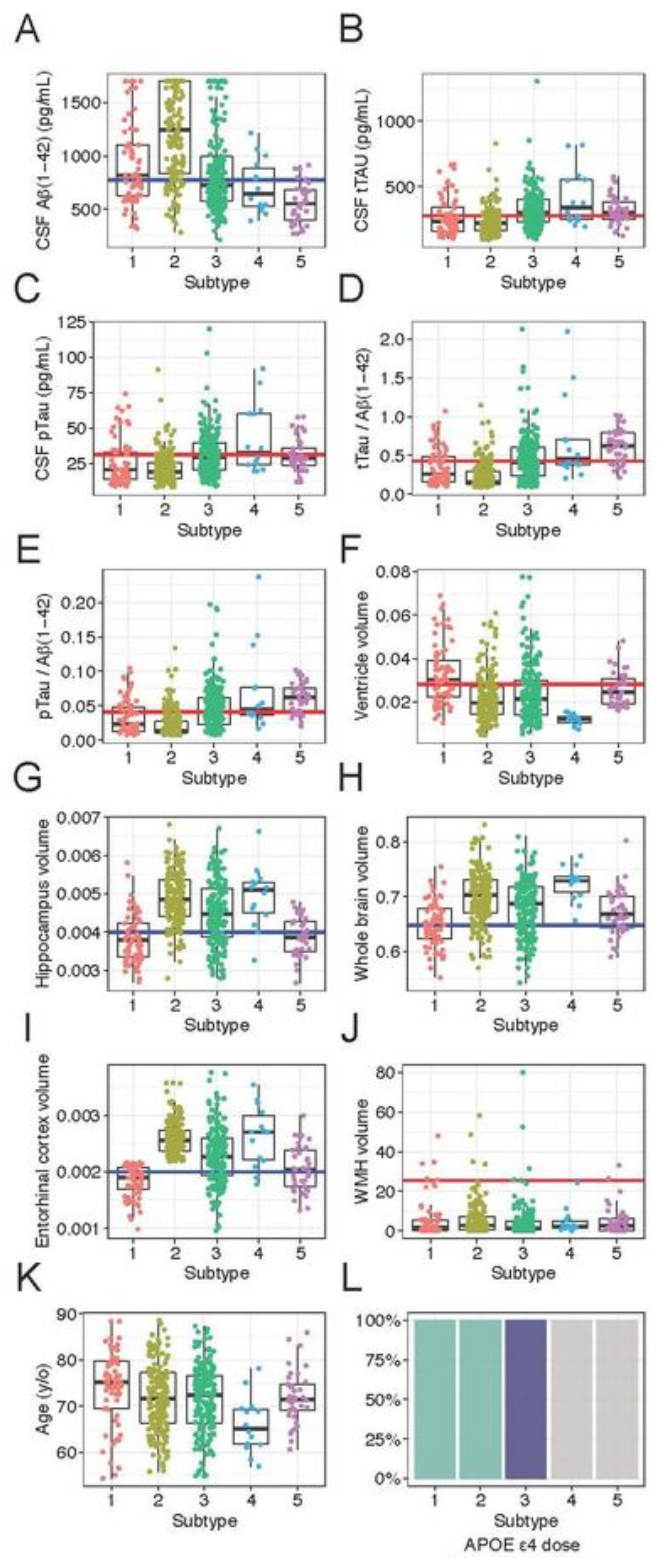

The cutoffs based on the $\mathrm{CNs}$ and the ADs.

- Higher value is closer to $A D=$ Lower value is closer to $A D$

\section{Figure 1}

Features in each subtype. (A) CSF A $\beta(1-42)$ level, (B) CSF tTau level, (C) CSF pTau level, (D) the tTau/A $\beta(1-42)$ ratio, (E) the pTau/A $\beta(1-42)$ ratio, (F) the normalized ventricular volume, $(G)$ the normalized hippocampal volume, $(\mathrm{H})$ the normalized whole-brain volume, $(\mathrm{I})$ the normalized entorhinal cortex volume, 
$(\mathrm{J})$ the normalized WMH volume, $(\mathrm{K})$ age, and $(\mathrm{L})$ APOE $\varepsilon 4$ alleles. The blue and red lines indicate the cutoff values based on the $\mathrm{CN}$ subjects and the $\mathrm{AD}$ dementia patients. Points below the blue line or above the red line represent levels similar to those in $A D$ dementia patients.

\section{Figure 5}
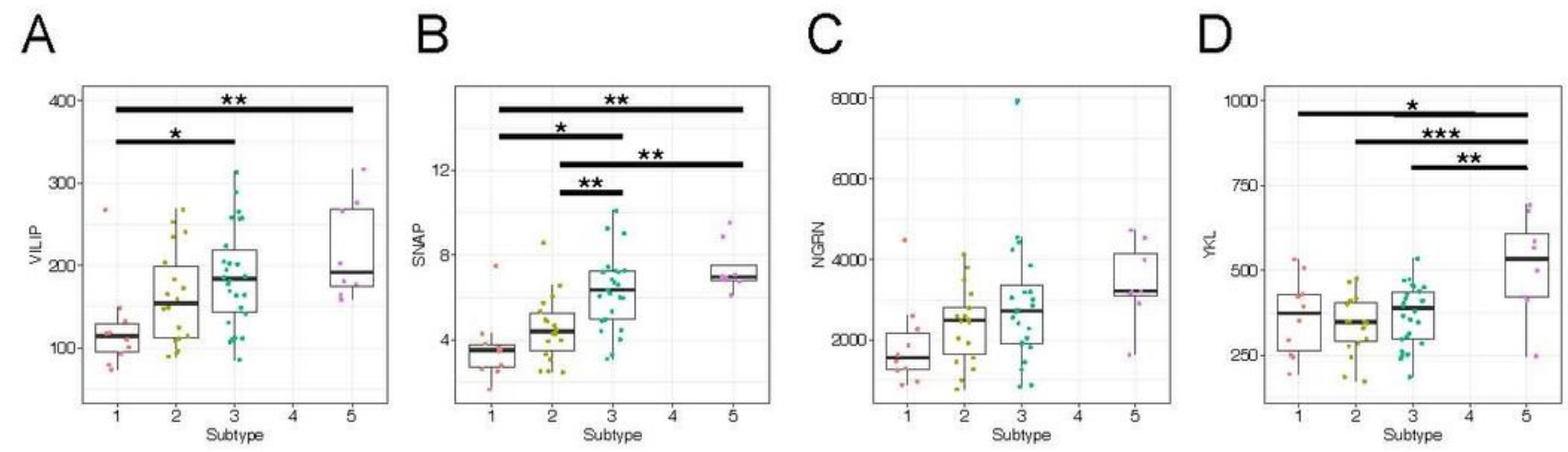

\section{Figure 1}

Levels of the CSF neuronal and synaptic injury markers VILIP-1 (A), SNAP-25 (B), and NGRN (C) and the inflammatory response marker YKL-40 (D). The Tukey's HSD test was used to verify the differences in CSF levels between two subtypes and was applied as a multiple comparison procedure. ${ }^{*} p<0.05$, ${ }^{*} p<$ $0.01, * \star * p<0.001$. 


\section{Figure 6}

A

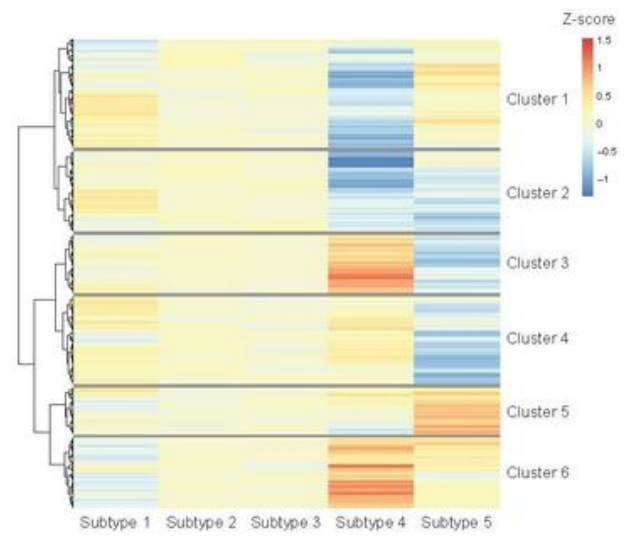

B
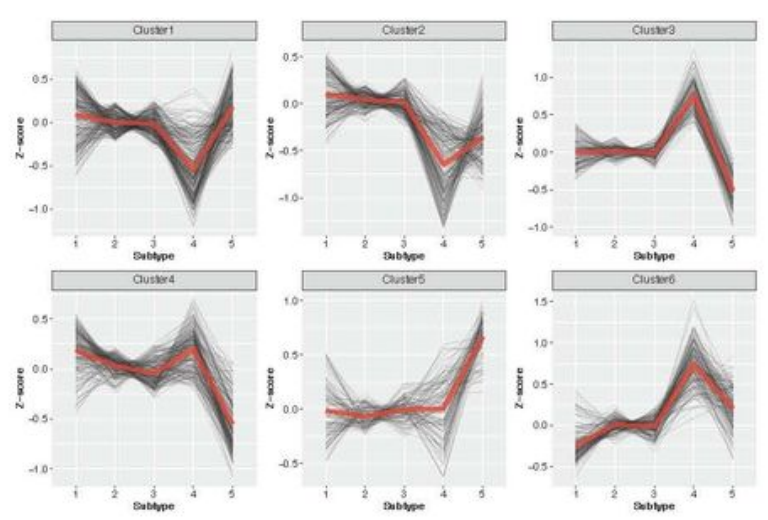

C
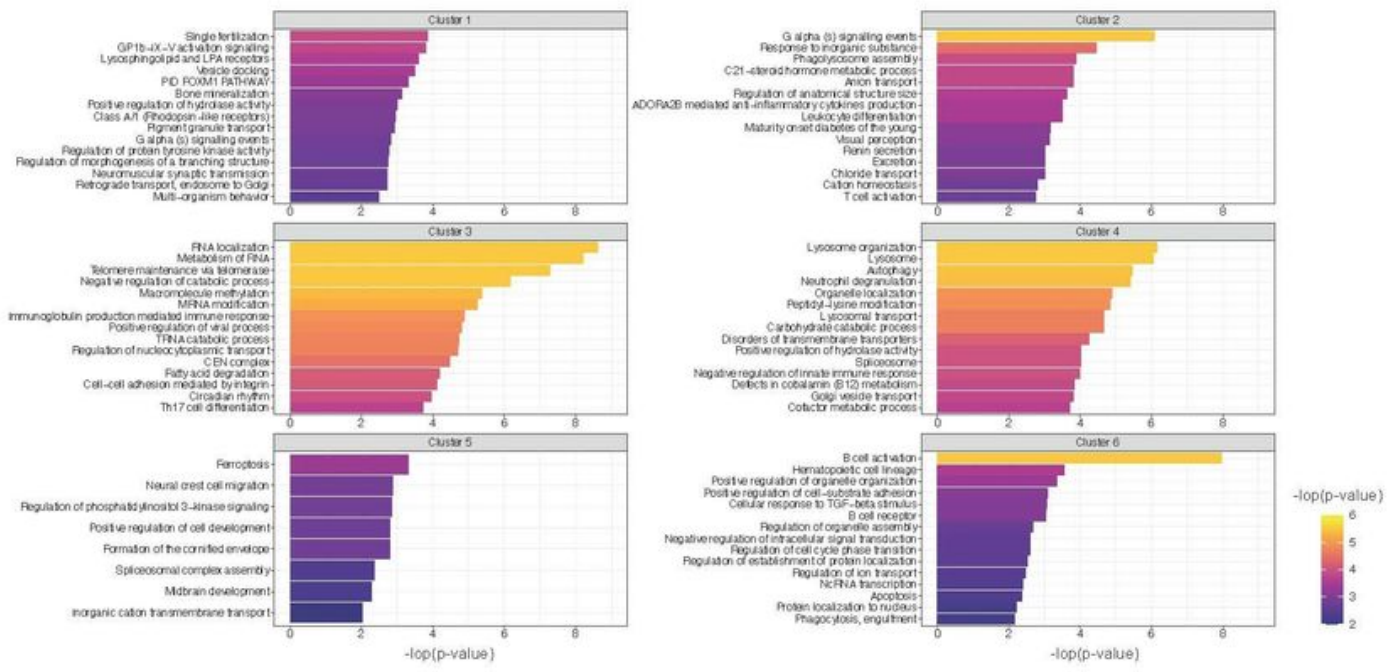

$\mathrm{E}$
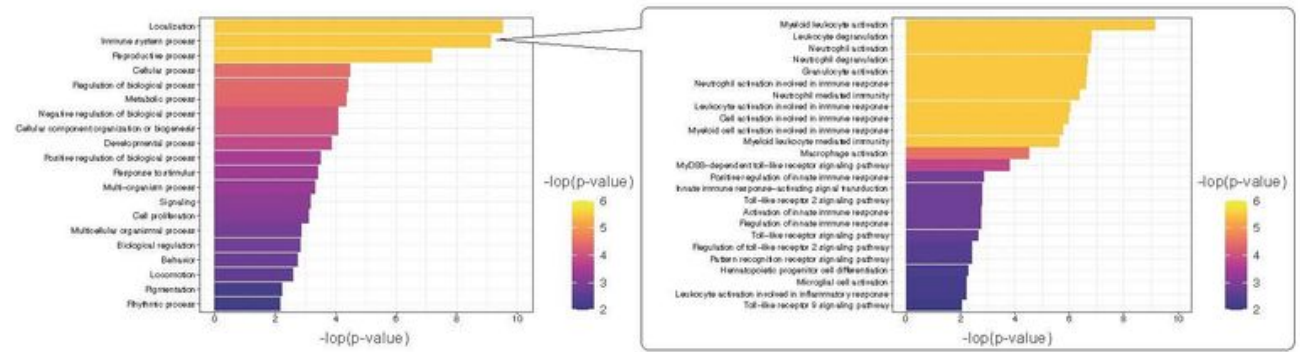

\section{Figure 1}

Gene expression analysis using peripheral blood samples. (A) Heatmap and hierarchical clustering of 1,080 DEGs. The colour represents the normalized gene expression level (z-score). Each row represents each gene. Clustering was performed via Ward's method. The number of clusters was set to six based on the gap statistic (Figure S4). (B) Gene expression profiles of the six clusters. The black and red polygonal lines represent the z-scores of the genes and the averaged z-scores, respectively. (C) Gene functional 
enrichment analysis of the genes in each cluster. Each bar shows the significances of the enriched terms for the genes in each cluster. (D, E) Parent terms (D) and immune system pathways (E) of the genes that were correlated with the inflammation marker YKL-40. The bars in $(E)$ indicate the enrichment of terms with the parent term "Immune system process".

\section{Supplementary Files}

This is a list of supplementary files associated with this preprint. Click to download.

- Supplementaryinformation.docx 